\title{
EL USO DE RAZONAMIENTOS CAUSALES EN RELACIÓN CON LA SIGNIFICATIVIDAD DE LOS MODELOS TEÓRICOS
}

\author{
SOLSONA PAIRÓ, NÚRIA ${ }^{1}$, IZQUIERDO AYMERICH, MERCÈ ${ }^{2}$ y GUTIÉRREZ, RUFINA ${ }^{3}$ \\ ${ }^{1}$ IES Josep Pla. Barcelona. \\ ${ }^{2}$ Universitat Autònoma de Barcelona. Departament de Didàctica de les Ciències \\ Experimentals i les Matemàtiques. \\ ${ }^{3}$ Instituto de Estudios Pedagógicos Somosaguas (IEPS). Departamento de Didáctica de las Ciencias \\ Experimentales. Madrid.
}

\begin{abstract}
SUMMARY
This paper presents the development and results of a part of one more general research about chemical change. It points out the use of the causality when students explain one phenomenon with two chemical changes called Copper cycle. The results of our study show the specific characteristics of the use of the causality in chemistry, and its relation with the theoretical models and chemical entities constructed by students along the learning of the concept of chemical change at the end of Senior High School in Barcelona.
\end{abstract}

\section{INTRODUCCIÓN}

Los resultados obtenidos en investigaciones realizadas en el campo de didáctica de la química permiten afirmar que la construcción del concepto de cambio químico en la escuela secundaria es uno de los objetivos centrales a abordar durante el proceso de aprendizaje. Se han detectado dificultades en el aprendizaje de los conceptos químicos (Solomonidou, 1991; Llorens, 1991, Roletto, 1994; Martín, 1994; Ramsden, 1997; Stavridou, 1998), se ha analizado la evolución de las explicaciones a diferentes edades (Prieto, 1993) y se han identificado modelos de explicación en el alumnado (Andersson, 1990; Sanmartí, 1995; Watson, 1995).

Un razonamiento causal es aquél que se caracteriza por asociar «causas» $\mathrm{y}$ «efectos» a los fenómenos que acontecen. Una concepción causal espontánea responde a una determinada visión del mundo, a un modo de ver y explicar cómo se desarrollan los acontecimientos, y está presente en la manera habitual de expresarse las personas y en los mecanismos de acción que describen. En el período de iniciación en aprendizaje de la química adquiere mucha importancia el entrenamiento del alumnado en la elaboración de las explicaciones químicas y es probable que en este momento el alumnado construya sus explicaciones utilizando un razonamiento causal o un razonamiento analógico.

En ocasiones, el aprendizaje tiene lugar al identificar cada nuevo concepto o fenómeno como si se pareciera a algo que ya conocemos previamente. En varias investigaciones se ha detectado el uso de analogías en diferentes situaciones de aprendizaje (Duit, 1991; Thagard, 1992; Ogborn, 1996; Treagust et al., 1996). El uso del pensamiento analógico presenta limitaciones en el apren- 
dizaje del concepto de cambio químico, ya que el alumnado no puede relacionar este concepto con nada que conozca previamente, y hemos observado que, cuando se utilizan analogías, funcionan más para decir lo que no es el cambio químico que no para elaborar una explicación sobre el mismo (Solsona, 1997).

Entre los estudios realizados dentro del marco constructivista hay pocas investigaciones empíricas directas sobre el uso de la causalidad (Pozo, 1991). La investigación que presentamos en este artículo sobre el estudio del uso del razonamiento causal forma parte de una investigación más amplia que aborda el aprendizaje de la interpretación de los fenómenos químicos (Solsona, 1997). El estudio del uso de los razonamientos causales en el análisis de las entrevistas es una primera aplicación, en el ámbito de la química, del análisis de la causalidad, hecho por Rufina Gutiérrez (1994), en física, según el modelo de De Kleer y Brown. Gutiérrez (1994) señala que en muchas ocasiones, en la investigación didáctica, ante un conocimiento explícito determinado se interpreta que proviene de un determinado conocimiento implícito del alumno. Antes de hacer esta interpretación habría que preguntarse de dónde procede esta explicación y dar oportunidad al alumno a que explicite este conocimiento implícito.

En nuestro trabajo de investigación abordamos las dificultades específicas que se presentan en el aprendizaje del concepto de cambio químico, tanto en sus componentes factuales como teóricos; es decir, tanto de los hechos como de los conceptos químicos. Además, analizamos cómo se llega a la idea de que algo no cambia, el elemento químico, viendo que cambian las sustancias.

Se ha insistido repetidamente en que la enseñanza de las ciencias debe ser experimental, pero también se ha criticado la falta de sentido global de los experimentos escolares para el alumnado y su desvinculación de los conceptos teóricos que se trabajan (White, 1996). En clase de química, es necesario que el alumnado aprenda a mirar los fenómenos de la manera específica que lo hace la química y para ello debe ser capaz de construir explicaciones de los fenómenos utilizando los conceptos químicos.

\section{UN ENFOQUE DESDE LAS CIENCIAS COGNITIVAS}

Desde las ciencias cognitivas, se propone que el conocimiento científico se construya mediante el establecimiento de una relación de similitud entre un hecho y el modelo teórico que lo interpreta, de manera que lleguen a contenerse mutuamente y formen una teoría (Giere, 1988; Izquierdo, 1996). Cuando un hecho se convierte en un «hecho interpretado», puede llegar a funcionar como un modelo teórico respecto a otros hechos que aún no se han interpretado. En nuestra opinión, los modelos teóricos que va construyendo el alumnado durante el aprendizaje escolar, como resultado de la intervención docente, tienen en gran parte carácter implícíto. Estos modelos teóricos le permiten explicar los fenómenos y razonar a partir de los experimentos, y pueden evolucionar a lo largo de la escolarización.

El problema aparece cuando debe introducirse un hecho nuevo que requiere un nuevo modelo, como el de cambio químico, puesto que es difícil llegar a captar la similitud entre él y otros fenómenos interpretados implícitamente con anterioridad, mediante el modelo «cambio de estado» o simplemente considerándolo un fenómeno «natural». En esta situación, lo importante es llegar a interpretar el fenómeno; cuando esto se consigue, el fenómeno adquiere las características del modelo y podrá funcionar como analogía para explicar otro fenómeno desconocido del mismo tipo.

Dado que el alumnado tiene acceso a un número de hechos o fenómenos más reducido de los que intentan explicar las teorías científicas propias de cada una de las disciplinas, los modelos teóricos serán simples al inicio del aprendizaje y se harán más complejos a medida que se conozcan más hechos que explicar. Se trata de superar las explicaciones adecuadas sólo para un pequeño numero de fenómenos.

La construcción de los modelos teóricos requiere dar sentido a las situaciones experimentales que se planteen al alumnado y han de permitir razonar a partir de las mismas. Por ello, en situación de aprendizaje identificamos los modelos teóricos que utiliza el alumnado a través del conjunto de explicaciones que es capaz de elaborar sobre uno o diversos hechos interpretados.

\section{UNA INVESTIGACIÓN SOBRE LA EMER- GENCIA DEL CONCEPTO DE CAMBIO QUIIMICO}

Hemos llevado a cabo una investigación amplia sobre el concepto de cambio químico, a lo largo de tres años, de $2^{\circ}$ de BUP a COU, con un grupo de 51 chicos y chicas de un instituto de BUP de Barcelona (Solsona, 1997). Durante la investigación realizada en contexto escolar se utilizaron varios instrumentos, entre ellos la confección de una redacción, un cuestionario sobre la realización del experimento llamado ciclo del $\mathrm{Cu}$ (Solsona e Izquierdo, 1998b) y una entrevista sobre el mismo experimento.

Para el análisis de la redacciones se establecieron unas categorías que permitieron detectar que el alumnado habla sobre el cambio químico a partir de tres diferentes enfoques globales que llamamos: modelo mecano, cocina e interactivo. En cada uno de los modelos, los conceptos utilizados para explicar el cambio químico, las relaciones que se establecen entre los conceptos y los ejemplos que se dan de cambio químico presentan regularidades en un mismo modelo y diferencias entre los modelos. Un cuarto grupo de alumnos y alumnas no llegó a formular ningún modelo y los hemos reunido en un último grupo que llamamos modelo incoherente (Solsona e Izquierdo, 1998a; Izquierdo y Solsona, 1999). 
El modelo mecano de cambio químico es aquél en el que el discurso se construye fundamentalmente en torno a la explicación microscópica del cambio, sin dar importancia a los fenómenos. Se habla del cambio desde la estructura interna, los ejemplos son teóricos o en algunos casos no se indican ejemplos y es habitual el uso de reglas analógicas implícitas.

El modelo cocina de cambio químico es aquél en el que el discurso se construye fundamentalmente en torno a los fenómenos. En el texto escrito se citan uno o dos ejemplos empíricos, a partir de los cuales se infiere qué es un cambio químico, y se explica como un cambio físico o un cambio de propiedades. Los ejemplos de cambio que se citan también son empíricos.

El modelo interactivo de cambio químico es aquél en el que la interpretación del cambio químico se realiza en términos de cambio de sustancias, es decir, de formación de nuevas sustancias que sustituyen claramente las sustancias iniciales. En la interpretación del cambio y, por lo tanto, en la construcción del discurso hay una relación coherente y equilibrada entre el nivel de explicación macroscópico y microscópico. Los ejemplos que se citan son teóricos y el texto es coherente globalmente.

El modelo incoherente del cambio químico es aquél en el que no se explica el cambio químico, los ejemplos que se citan del mismo tienen carácter ilustrativo y no están razonados. En él no se utiliza claramente ninguna terminología, ni macroscópica, ni microscópica para describirlo. El texto escrito sobre el cambio químico se construye sin una progresión temática ordenada, en forma de islas de conocimiento, sin relación entre ellas. El alumnado presenta, en la mayoría de los casos, una lista sin conexión de lecciones de cosas y de definiciones. En este caso, el alumnado no ha sabido integrar sus propias ideas con las que se han trabajado en clase.

Cuadro I

Número de alumnos y alumnas en cada modelo teórico.

\begin{tabular}{|l|c|c|}
\hline $\begin{array}{c}\text { Modelo } \\
\text { cambio químico }\end{array}$ & $\begin{array}{c}\text { Núm. total } \\
\text { alumnado }\end{array}$ & $\begin{array}{c}\text { Porcentaje } \\
\text { alumnado }\end{array}$ \\
\hline Modelo incoherente & 10 chicas, 7 chicos $=17$ & $33 \%$ \\
\hline Modelo mecano & 6 chicas, 11 chicos $=17$ & $33 \%$ \\
\hline Modelo cocina & 4 chicas, 9 chicos $=13$ & $26 \%$ \\
\hline Modelo interactivo & 1 chica, 3 chicos $=4$ & $8 \%$ \\
\hline
\end{tabular}

El cuadro I recoge el número de alumnas y alumnos agrupados en cada uno de los modelos teóricos.

El alumnado agrupado en el modelo interactivo ha interiorizado el modelo científicamente aceptado de cambio químico, pero es dudoso que esto haya sucedido en los otros tres modelos: mecano, cocina e incoherente.
Los modelos teóricos de cambio químico construidos durante nuestra investigación a partir del contenido de las producciones escritas del alumnado, ya sean las respuestas a cuestionarios o las redacciones, son en realidad fotografías estáticas de un pensamiento dinámico. Debido fundamentalmente a la naturaleza dinámica de los datos y a los resultados obtenidos en las pruebas anteriores, vimos la necesidad de intentar ver si el alumnado podía comunicar mejor sus explicaciones con las entrevistas.

\section{DINÁMICA DEL RAZONAMIENTO EN LAS EXPLICACIONES DE LOS ALUMNOS}

Para la investigación de la naturaleza dinámica del razonamiento, durante el tercer año de la investigación, una muestra de 12 estudiantes, 5 chicos y 7 chicas, fue entrevistada personalmente sobre el experimento llamado ciclo del Cu (Andersson, 1990; Llorens, 1991), que incluye las dos reacciones siguientes:

$$
\begin{aligned}
& \mathrm{Cu}+4 \mathrm{HNO}_{3} \rightarrow \mathrm{Cu}\left(\mathrm{NO}_{3}\right)_{2}+2 \mathrm{NO}_{2}+2 \mathrm{H}_{2} \mathrm{O} \\
& \mathrm{Cu}\left(\mathrm{NO}_{3}\right)_{2}+\mathrm{Fe} \rightarrow \mathrm{Fe}\left(\mathrm{NO}_{3}\right)_{2}+\mathrm{Cu}
\end{aligned}
$$

El alumnado ya había realizado el experimento del ciclo del $\mathrm{Cu}$ dos veces con anterioridad a la entrevista, en $2^{\circ} \mathrm{y}$ $3^{\circ}$ de BUP, con un año de diferencia. La entrevista se desarrolló siguiendo una pauta orientativa que contenía preguntas relativas a la segunda reacción incluida en el ciclo del $\mathrm{Cu}$ que se realizó in situ.

Las entrevistas fueron pensadas para acercarnos por aproximaciones sucesivas a las explicaciones del alumnado y para evitar una interpretación prematura o precipitada de los datos obtenidos hasta entonces en la investigación. Para ello intentamos que las entrevistas transcurrieran en un tono de conversación que permitiera la libre expresión de la persona entrevistada y facilitara la libre expresión de su pensamiento espontáneo, sin restricciones. Con este objetivo tratamos de que el control del diálogo lo llevara tanto la persona entrevistada como la entrevistadora; es decir, nuestro objetivo no era que la persona entrevistada nos diera la explicación científica «verdadera» sino que nos diera su explicación.

El objetivo de la entrevista es identificar el uso del razonamiento causal que aparece en la explicación de la reacción entre el $\mathrm{Cu}\left(\mathrm{NO}_{3}\right)_{2}$ y el Fe. Nuestra hipótesis de trabajo, de acuerdo con Rufina Gutiérrez (1994) es que las personas, cuando pensamos espontáneamente, utilizamos estructuras causales para explicar los fenómenos identificados. Hemos querido averiguar si existe alguna relación entre los diferentes modelos teóricos identificados en las redacciones escritas por el alumnado y el uso de la causalidad.

La entrevista se centró en la reacción entre el $\mathrm{Cu}\left(\mathrm{NO}_{3}\right)_{2}$ y el Fe, con el intento de dar más oportunidades para que el alumnado explicitara su interpretación del ciclo del cobre. Tanto la primera reacción $\left(\mathrm{Cu}+4 \mathrm{HNO}_{3} \rightarrow\right.$ 
$\left.\mathrm{Cu}\left(\mathrm{NO}_{3}\right)_{2}+2 \mathrm{NO}_{2}+2 \mathrm{H}_{2} \mathrm{O}\right)$ como la segunda reacción del ciclo del $\mathrm{Cu}:\left(\mathrm{Cu}\left(\mathrm{NO}_{3}\right)_{2}+\mathrm{Fe} \rightarrow \mathrm{Fe}\left(\mathrm{NO}_{3}\right)_{2}+\mathrm{Cu}\right)$ habían presentado problemas, en los aspectos fenomenológicos y en la interpretación teórica. Pero había algunas respuestas del alumnado a la segunda reacción que no quedaban claras. Por ello se centró la entrevista en la segunda reacción: el $\mathrm{Cu}\left(\mathrm{NO}_{3}\right)_{2}$ con el Fe.

En la pauta orientativa para realizar la entrevista, las reglas de oro son $a$ ) no hablar más que las personas entrevistadas, no plantear preguntas inductoras, hacer preguntas abiertas, no dicotómicas, simples y que impliquen una sola idea; $b$ ) no introducir nuevos conceptos y no añadir información a la que da la persona entrevistada. En la confección de las preguntas utilizadas en nuestra entrevista hemos seguido dos criterios: utilizar las propias palabras del alumnado, siempre que fuera posible, para sugerirles que expliquen mejor sus afirmaciones, y utilizar preguntas abiertas y simples, pero que hicieran reflexionar sobre el experimento.

En un primer momento combinamos preguntas de definición (¿Qué es para ti un ion?) con preguntas de comparación (¿Qué relación de semejanza o de diferencia crees que hay entre un ion y un átomo?) Pero, en las últimas entrevistas, estas preguntas no se plantearon, porque inducían respuestas memorísticas o de libro, que no permitían acercarnos al modelo de explicación del experimento construido por cada chico o chica y, por lo tanto, no han sido utilizadas en el análisis.

En cuanto a la administración de la entrevista, el establecimiento de la temática sobre la que se iba a desarrollar fue relativamente fácil, puesto que el alumnado sabía que la entrevistadora era la misma persona que en cursos anteriores les había propuesto la realización de diferentes pruebas para averiguar sus ideas sobre el cambio químico, y la conocían personalmente. De esta forma, con unas breves palabras, la entrevistadora concertaba los términos y el tema de la entrevista con el objetivo de comprender mejor las explicaciones que había recibido en los cuestionarios escritos realizados anteriormente. Constatamos la satisfacción que reflejaban las chicas y los chicos por el hecho de ser entrevistados.

\section{ANÁLISIS DE LAS ENTREVISTAS}

En el análisis de las producciones del alumnado, habíamos observado la facilidad de éstos en utilizar simultáneamente diferentes tipos de explicaciones para un mismo fenómeno. Por ejemplo, si se comparan las respuestas dadas al cuestionario que acompaña la realización del experimento del ciclo del cobre, con anterioridad a la entrevista, se observa la facilidad con que una misma persona puede cambiar de explicación, poniendo o quitando elementos, que tanto considera centrales en la explicación de un fenómeno, como, de pronto, considera secundarios.

Abordamos el análisis del razonamiento causal implícito en las explicaciones elaboradas en las entrevistas porque era la primera oportunidad que teníamos para interrogar al alumno o alumna respecto a las acciones que está realizando sobre el fenómeno. Los anteriores instrumentos de investigación no proporcionaban información sobre su actuación acerca del fenómeno químico o sistema objeto de estudio.

Las categorías que hemos utilizado en el análisis de las entrevistas coinciden con algunas de las categorías utilizadas en el análisis de las redacciones. Las unidades de análisis que recogen las distintas categorías de las entrevistas son las siguientes: ejemplos de cambio químico, qué cambia, qué se conserva durante el cambio, nivel de explicación del cambio, uso del razonamiento analógico y uso de la causalidad por parte del alumnado en el momento de elaborar las explicaciones. En este artículo vamos a referirnos únicamente a aquellas categorías que aparecen en la investigación relacionadas con el uso de la causalidad en el caso del ciclo del $\mathrm{Cu}$, que son las que aparecen en el cuadro II que hay a continuación.

Cuadro II

Ejemplo de las categorías de análisis en la entrevista realizada con Santi.

\begin{tabular}{|c|l|}
\hline Categoría & Unidades de análisis seleccionadas \\
\hline Qué cambia & $\begin{array}{l}\text { (5) «[...] teníamos el nitrato de cobre en forma } \\
\text { iónica; por un lado, teníamos el cobre y, por otro, } \\
\text { el nitrato y, al poner el hierro, el cobre se ha } \\
\text { depositado alrededor del hierro y un poco de } \\
\text { hierro del clavo se ha extendido y se ha hecho } \\
\text { nitrato de hierro». }\end{array}$ \\
\hline $\begin{array}{l}\text { Qué se } \\
\text { conserva }\end{array}$ & $\begin{array}{l}\text { (13) «[..] los átomos de los elementos, los } \\
\text { núcleos o los átomos, lo que cambia es que se } \\
\text { juntan de una forma o forman otro compuesto». }\end{array}$ \\
\hline $\begin{array}{l}\text { Nivel de } \\
\text { explicación }\end{array}$ & $\begin{array}{l}\text { (5) + (6) «[...] en un principio hay un equilibrio, } \\
\text { al poner el clavo de hierro, de los átomos que se } \\
\text { desprenden del hierro; hay un equilibrio de los } \\
\text { que se desprenden con los que se juntan». }\end{array}$ \\
\hline $\begin{array}{l}\text { Uso de la } \\
\text { causalidad }\end{array}$ & $\begin{array}{l}\text { (5), (6) (7) «[...] pero al calentarlo, aceleramos la } \\
\text { reacción y se acelera el proceso de los átomos de } \\
\text { hierro que se liberan del sólido y pasan a forma } \\
\text { iónica», (8) «[...] lo que hacia el hierro es dar } \\
\text { electrones de su átomo y el cobre coge los } \\
\text { electrones. Hay una transferencia de electrones de } \\
\text { su átomo el cobre coge los electrones. Hay una } \\
\text { transferencia de electrones que hace que el nitrato } \\
\text { de Cu pase a nitrato de Fe», (9) «[...] los } \\
\text { compuestos no están formando una molécula, } \\
\text { sino que el cobre está en forma iónica; un catión } \\
\text { que ha perdido electrones y está en disolución en } \\
\text { la sustancia y no se ha unido al anión, que es el } \\
\text { nitrato que está con carga negativa, porque ha } \\
\text { ganado electrones», (10) «[...] para saber si } \\
\text { tenemos cationes y aniones hacemos pasar } \\
\text { electricidad,... por atracción de cargas», (11.1) } \\
\text { redox [...] hay una sustancia que pierde electrones } \\
\text { y otra que los gana, el cobre es el que gana los } \\
\text { electrones, es decir, pasa de tener carga +2 a tener } \\
\text { carga 0, porque deposita en forma sólida y el } \\
\text { hierro es sólido, o sea, está a cero y pasa a +2». }\end{array}$ \\
\hline
\end{tabular}


Los números entre paréntesis indican las unidades de análisis tal como se numeraron en la transcripción de la entrevista.

En nuestro estudio hemos identificado cuatro tipos de estructuras causales. Las «estructuras causales lineales» son aquéllas que tienen un agente causal, tal como puede verse en la figura 3. Las «cadenas causales» son aquéllas que incluyen dos explicaciones o estructuras causales, con la actuación de dos agentes causales, de forma que el efecto producido en la primera estructura causal actúa de agente causal de una segunda estructura que va encadenada a la primera, tal como puede observarse en la figura 4. Las dos estructuras, tanto la lineal como la cadena causal pueden ser simples o complejas según produzcan uno o varios efectos o materiales finales.

En nuestro estudio, 5 alumnos y 4 alumnas usan estructuras lineales causales y 4 alumnos y 3 alumnas usan cadenas causales, tal como puede verse en la figura 4.

Para el análisis del razonamiento causal hemos detectado que el alumnado utiliza los siguientes elementos que constituyen cada estructura causal: los materiales iniciales, los materiales finales, la acción llevada a cabo por un agente causal y el proceso que tiene lugar en el sistema. Por ejemplo, Santi dice: «[...] teníamos el nitrato de cobre en forma iónica; por un lado, teníamos el cobre y, por otro, el nitrato y, al poner el hierro, el cobre se ha depositado alrededor del hierro y un poco de hierro del clavo se ha extendido y ha formado nitrato de hierro».

A partir de la interpretación de las frases del alumno construímos una descripción funcional en la que identificamos como materiales iniciales del sistema el Fe, el $\mathrm{Cu}^{2+}$ y el $\mathrm{NO}_{3}{ }^{-}$. La acción es la de «poner el hierro»y los materiales finales del sistema son $\mathrm{Cu}$ y $\mathrm{Fe}\left(\mathrm{NO}_{3}\right)_{2}$.

La descripción funcional de esta estructura causal lineal compleja de Santi es la siguiente (Cuadro III):

Cuadro III

Descripción de una estructura causal lineal compleja.

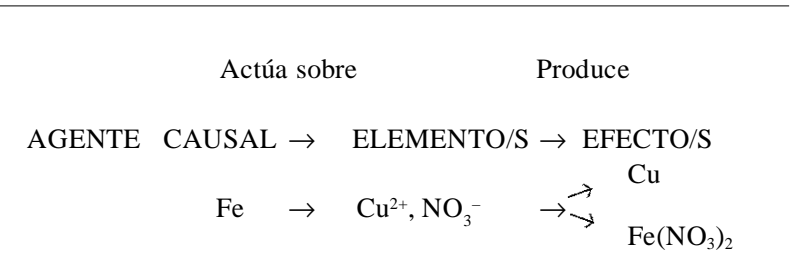

La estructura causal anterior es lineal aunque compleja porque un solo agente causal produce dos efectos.

En otras ocasiones, el alumnado utiliza cadenas causales, en las que el efecto producido en una primera estructura causal actúa como agente causal de una se- gunda estructura que va encadenada a la primera. Así, Santi dice «[...] en un principio, hay un equilibrio, al poner el hierro de los átomos que se desprenden del hierro con los que se juntan; pero, al calentarlo, aceleramos la reacción, y se acelera el proceso de los átomos de Fe que se liberan del sólido y pasan a forma iónica».

Los materiales iniciales de la primera estructura causal son el Fe, el $\mathrm{Cu}^{2+}$ y el $\mathrm{NO}_{3}{ }^{-}$. La acción es la de «poner el hierro» y el material final del sistema es «el equilibrio de los átomos de Fe». Sobre este material obtenido como producto de la primera estructura causal actúa un segundo agente causal que es «el calor» y los materiales finales de la segunda estructura causal son «la reacción que se acelera entendida como proceso y los átomos de Fe que se liberan del sólido y pasan a forma iónica»

La descripción funcional de esta cadena causal compleja que incluye dos explicaciones es la que se describe en el cuadro IV.

\section{Cuadro IV}

Descripción de la cadena causal compleja.

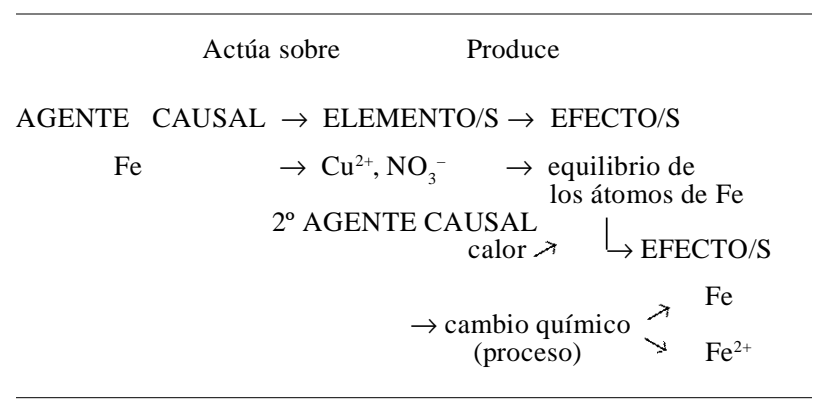

Queremos remarcar la importancia de aquel sector del alumnado que utiliza la causalidad procesual (Solsona, 1997). Tal como se puede ver en la figura 4, además de Santi, otros alumnos construyen estructuras causales en las que el cambio químico se explica como un proceso, es decir, como una sucesión de pasos encadenados que tienen lugar entre las sustancias que reaccionan, no como un fenómeno en el cual desaparecen unas sustancias y se obtienen otras nuevas sustancias. Por ejemplo, Elena construye una estructura de causalidad procesual cuando dice que «el calor, el $\mathrm{Cu}\left(\mathrm{NO}_{3}\right)_{2}$ ha hervido y esto hace que reaccione con el hierro, haciendo que se herrumbre».

Los agentes causales utilizados por el alumnado son elementos del sistema como el clavo, el ácido, el reactivo, el alambre, el $\mathrm{Cu}$ y el Fe. Pero también utilizan como agentes causales elementos externos del sistema como el calor, la acción de agitar, el calentador, la temperatura y la electricidad.

La estructura más utilizada en las explicaciones causales construidas en nuestra investigación es: agente causal $\rightarrow$ 
uno o más materiales $\rightarrow$ uno o más efectos, tal como se puede ver en el cuadro $\mathrm{V}$.

Santi es el único alumno que construye una cadena causal compleja con dos agentes causales. El resto de los que utilizan la causalidad construye cadenas causales en las que, en la mayoría de los casos, el agente causal actúa sobre dos o tres materiales iniciales y/o se obtienen dos materiales finales. Pero parece ser que ésta es una característica del razonamiento causal en química y habrá que verificar si se repite en otras investigaciones.

Las tres alumnas que no utilizan explicaciones causales, en nuestro estudio, se limitan a hacer descripciones o a repetir memorísticamente las explicaciones oídas en clase. No utilizan un razonamiento alternativo.

\section{LA CAUSALIDAD Y LOS MODELOS TEÓ- RICOS}

Una de las conclusiones más importantes de nuestro estudio se refiere a que el uso de explicaciones causales por parte del alumnado no depende del modelo teórico de cambio químico que tiene cada alumno.

Por ejemplo, frente a la pregunta «En la reacción entre el $\mathrm{Cu}\left(\mathrm{NO}_{3}\right)_{2}$ y el $\mathrm{Fe}$, ¿qué ha cambiado?», tanto Ainhoa, que tiene un modelo incoherente de cambio químico, en la entrevista, como Santi, que tiene un modelo interactivo, dan una explicación causal. Ainhoa intenta dar una explicación macroscópica de la reacción entre el $\mathrm{Cu}\left(\mathrm{NO}_{3}\right)_{2}$ y el Fe, construyendo la siguiente frase causal: «[...] con el calor, [el nitrato de cobre] ha llegado al punto de ebullición y ha hecho que el clavo de hierro se oxidara». Es decir, utiliza la falsa analogía del «hierro oxidado». A la misma pregunta, Santi, que tiene un modelo interactivo del cambio químico dice: «[...] teníamos el nitrato de cobre en forma iónica; por un lado, el cobre y, por otro, el nitrato, y, al poner el hierro, el cobre se ha depositado alrededor del hierro y un poco del hierro del clavo se ha extendido y ha formado nitrato de hierro».

Jordi, que se caracteriza por tener un modelo mecano de cambio químico antes de la entrevista, contesta de la siguiente forma: «[...] el Fe ha cambiado de compuesto porque se le ha añadido cobre». Ana, que ha sido agrupada en el modelo cocina de cambio químico dice: «[...] ha cambiado el color del clavo porque se ha oxidado al añadir calor».

A la vista de los resultados obtenidos, podemos afirmar que usan explicaciones causales alumnos y alumnas agrupados en los distintos modelos teóricos del cambio químico que hemos construido en la investigación, desde el interactivo hasta el incoherente. No se observa un comportamiento homogéneo en el uso de la causalidad en cada modelo concreto. Santi, que hemos agrupado en el modelo interactivo, construye 6 estructuras causales, 1 de ellas encadenadas; pero no ocurre lo mismo con Jose, Gemma y Marta, que también corresponden a un modelo interactivo: Jose construye 3 estructuras causa- les, pero Gemma y Marta no construyen ninguna, tal como hemos resumido en el cuadro V.

Se observa que Montse, con un modelo cocina de cambio químico, construye tantas estructuras causales como Santi, que corresponde al modelo interactivo de cambio químico, como Jordi, que corresponde al modelo mecano, y Ainhoa, del modelo incoherente. Esto es posible porque, para cada cual, su modelo teórico, incluso en el caso del incoherente, tiene sentido, es operativo y coherente en sí mismo.

\section{LAS ENTIDADES QUÍMICAS QUE CONS- TRUYE EL ALUMNADO}

Para elaborar una explicación científica del cambio químico, el alumnado debe aprender a identificar las «entidades» (Ogborn et al., 1996) que forman parte del modelo teórico de cambio químico científicamente aceptado y a relacionar significativamente estas entidades con los fenómenos que observa. Las «entidades» químicas son constructos que sólo tienen sentido en el marco de la ciencias y, al no ser intuitivas, el alumnado debe aprenderlas durante el proceso de aprendizaje.

Las entidades usadas por el alumnado en las respuestas a la entrevista son de dos tipos: elementos del propio sistema que se analiza, como el $\mathrm{Cu}$, el ácido o el clavo de $\mathrm{Fe}$, y elementos externos al sistema, como el calor, la electricidad o un imán. Constatamos en la elaboración de explicaciones por el alumnado, y nos parece muy significativo, el uso polisémico del término cobre, que es utilizado con tres significados diferentes: el objeto de $\mathrm{Cu}$, la sustancia $\mathrm{Cu}$, y el cobre ion.

El cuadro V resume las entidades que utiliza el alumnado en las estructuras causales que construye.

El alumnado agrupado en el modelo teórico mecano utiliza explicaciones causales pero hace referencia a un solo tipo de entidad. Habla de «la composición del clavo» o del « $\mathrm{Cu}$ » como sustancia. Este alumnado, que no es capaz de relacionar significativamente las entidades químicas, intenta explicar, por ejemplo, las reacciones «redox» diciendo indistintamente que en éstas se ganan o pierden electrones, tal como pudimos recoger en las entrevistas.

Ainhoa, que hemos agrupado en el modelo teórico incoherente de cambio químico, utiliza entidades que son elementos del sistema que constituye la estructura causal. Pero sólo habla de la entidad objeto: «el clavo de hierro». El alumnado agrupado en el modelo teórico cocina hace referencia por lo menos a dos entidades: habla del $\mathrm{Cu}$ y el $\mathrm{Fe}$ como objeto y del «Cu disuelto».

El alumnado agrupado en el modelo teórico interactivo, como es el caso de Santi, utiliza las tres entidades a las que se puede hacer referencia al hablar de una sustancia: el objeto, la sustancia en sí y la sustancia en forma iónica. Así, Santi, refiriéndose al Cu habla del objeto («clavo de 
Cuadro V

Tipos de estructuras causales construidas y entidades utilizadas por el alumnado.

\begin{tabular}{|c|c|c|c|c|}
\hline Alumno & $\begin{array}{l}\text { Estructuras } \\
\text { causales } \\
\text { simples }\end{array}$ & $\begin{array}{l}\text { Estructuras } \\
\text { causales } \\
\text { complejas }\end{array}$ & $\begin{array}{l}\text { Entidades utilizadas que son } \\
\text { elementos del sistema }\end{array}$ & $\begin{array}{l}\text { Entidades o elementos } \\
\text { externos al sistema }\end{array}$ \\
\hline José & 2 & 1 & $\begin{array}{l}\text { Clavo, gas, líquido, redox, } \mathrm{Cu} \text {, } \\
\text { átomo } \mathrm{Fe} \text {, átomo } \mathrm{Cu}, \mathrm{Fe}\end{array}$ & $\begin{array}{l}\text { Calor (acción de) } \\
\text { agitar }\end{array}$ \\
\hline Javier & 4 & 1 (c.q. como proceso) & $\begin{array}{l}\text { Ácido, } \mathrm{Cu}, \mathrm{Fe} \text {, clavo de } \mathrm{Fe} \text {, } \\
\text { disol. de } \mathrm{Cu}, \mathrm{Cu} \text { desasociado }\end{array}$ & - \\
\hline Jordi & 6 & 1 (c.q. como proceso) & $\begin{array}{l}\text { Clavo, líquido, } \mathrm{Cu}, \mathrm{Fe} \text {, ácido, } \\
\mathrm{Cu} \text { depositado }\end{array}$ & Calentador \\
\hline Gemma & - & - & - & - \\
\hline Marta & - & - & - & - \\
\hline Montse & 7 & 1 (c.q. como proceso) & $\begin{array}{l}\text { Reactivo, tubo de } \mathrm{Cu} \text {, alambre } \\
\text { de } \mathrm{Cu} \text {, clavo, } \mathrm{Cu}\left(\mathrm{NO}_{3}\right)_{2}, \mathrm{Fe} \text {, } \\
\mathrm{Cu} \text { solidificado o cristalizado, } \\
\text { óxido, } \mathrm{Cu} \text { disuelto, sustancia }\end{array}$ & Temperatura \\
\hline Anna & $\begin{array}{c}5 \text { (1 c.q. } \\
\text { como proceso) }\end{array}$ & 1 (c.q. como proceso) & $\begin{array}{c}\text { Líquido, herrumbe, iones de } \mathrm{Fe}, \\
\text { Fe del clavo, átomos de } \mathrm{Cu}, \\
\mathrm{Cu} \text { sólido, iones de } \mathrm{Cu}, \\
\mathrm{Cu} \text { en disolución }\end{array}$ & Calentador, imán \\
\hline Raquel & 2 & - & Clavo de Fe & Calor \\
\hline Ainhoa & $\begin{array}{c}4 \text { ( } 1 \text { c.q. } \\
\text { como proceso) }\end{array}$ & 3 (c.q. como proceso) & $\begin{array}{c}\text { Clavo de } \mathrm{Fe} \text {, átomo de } \mathrm{Fe}, \\
\text { nitrato de } \mathrm{Cu} \text {, nitrato de } \mathrm{Fe}, \\
\text { capa de óxido, } \\
\text { átomo de } \mathrm{Cu}\left(\mathrm{NO}_{3}\right)_{2} \text {, líquido }\end{array}$ & $\begin{array}{l}\text { Calor, calentador } \\
\text { fuego }\end{array}$ \\
\hline Rosa & - & - & - & - \\
\hline Óscar & 1 & - & Electrones, ácido & - \\
\hline Santi & $\begin{array}{c}5 \text { (3 c.q. como } \\
\text { proceso) }\end{array}$ & 1 (c.q. como proceso) & $\begin{array}{c}\text { Clavo de } \mathrm{Fe}, \mathrm{Fe} \text { extendido, } \\
\text { Fe en forma iónica, átomos de } \mathrm{Fe} \\
\text { que se liberan del sólido, } \mathrm{Cu}, \\
\mathrm{Cu} \text { depositado, } \mathrm{Cu} \text { en forma } \\
\text { iónica, c.q. nitrato de cobre, } \\
\text { nitrato en forma iónica }\end{array}$ & $\begin{array}{l}\text { Calor, temperatura, } \\
\text { electricidad }\end{array}$ \\
\hline
\end{tabular}

$\mathrm{Fe}$ ); de la sustancia («el hierro extendido»); y de «átomos de Fe que se liberan del sólido»; y cuando habla del $\mathrm{Cu}$, se refiere al « $\mathrm{Cu}$ depositado», a la sustancia «cobre» $\mathrm{y} \ll$ al cobre en forma iónica».

\section{CONSIDERACIONES FINALES}

Nuestra investigación confirma que el razonamiento causal constituye una manera de pensar muy generaliza- 
da que el alumnado utiliza en el marco de diferentes modelos teóricos. No se puede relacionar el uso de explicaciones causales con la construcción de un determinado modelo teórico de cambio químico, tal como hemos explicado en el apartado que habla de la causalidad y los modelos teóricos.

Hemos detectado que utilizan este tipo de razonamiento aquellos alumnos y alumnas que tienen construidas unas entidades concretas en torno al cambio químico y, por lo tanto, las pueden representar mentalmente. En el alumnado agrupado en el modelo interactivo, las entidades, es decir, las sustancias que cambian (la oxidación, el elemento que se conserva) son coincidentes con las entidades de la química. En cambio, en el modelo incoherente de cambio químico, Ainhoa no reconoce las sustancias que intervienen en el cambio químico y, sin embargo, usa entidades imaginadas, como el objeto de «hierro oxidado», que no está presente, ya que lo confunde con el Cu depositado pero lo imagina mentalmente como tal. Por lo tanto, sí se puede relacionar el uso de un modelo teórico científicamente válido con el uso de las entidades adecuadas, como hace Santi, en el modelo interactivo.

Otra de las conclusiones de nuestra investigación en lo que se refiere al uso de explicaciones causales en química es que ésta reúne algunas características diferentes que no aparecen en el razonamiento causal investigado en física. Por ejemplo, en lugar de razonar en términos de una diversidad de causas, el alumnado razona hablando de unos pocos agentes que en nuestro estudio son agen-

\section{REFERENCIAS BIBLIOGRÁFICAS}

ANDERSSON, B. (1990). Pupils' Conceptions of Matter and its Transformations (age 12-16). Studies in Science Education, 18 , pp. 53-85.

DUIT, R. (1991). On the Role of Analogies and Metaphors in Learning Science. Science Education, 75(6), pp. 649-672.

GIERE, R. (1988). Explaining Science. A cognitive approach. Chicago: Univ. Chicago Press.

GUTIÉRREZ,R.(1994). Coherencia del pensamientoespontáneo y causalidad. El caso de la dinámica elemental. Tesis doctoral. Madrid: Universidad Complutense.

IZQUIERDO, M. (1996). Cognitive models of science and the teaching of science, history of sciences and curriculum. Proceedings of the Second Ph. D. Summer School. Tesalónica: Art of Text.

IZQUIERDO, M. y SOLSONA, N. (1999). The case of teaching and learning the concept of chemical change. Proceedings of ICASE-University Utrecht Symposium. tes materiales (el calor o la electricidad) que ponen en marcha un proceso o causan un efecto determinado: el cambio o la reacción. Creemos que puede ser debido a las dificultades que el alumnado encuentra para familiarizarse con la química, mientras que los fenómenos mecánicos estudiados por Gutiérrez le resultan más próximos.

En nuestros resultados, el alumno prioriza el uso de explicaciones causales de manera paralela a la capacidad de intervención y de implicación en el fenómeno, con el conocimiento descriptivo de las sustancias o el interés por la química descriptiva.

Los resultados de las entrevistas nos han permitido comprender mejor el uso por parte del alumnado de los modelos de cambio químico y de las entidades que utiliza en la elaboración de sus explicaciones. La entrevista confirma los criterios de agrupación del alumnado en los modelos mecano y cocina y ha permitido ampliar la información sobre el uso de estos modelos.

Los resultados obtenidos en nuestra investigación en cuanto al uso de explicaciones causales con independencia de los modelos elaborados sobre el cambio químico tienen consecuencias para el proceso de enseñanza y aprendizaje, puesto que, si esta forma de razonamiento es utilizada espontáneamente por la mayor parte del alumnado, debe ser tenida en cuenta por el profesorado y potenciada como estrategia que favorezca la construcción de un modelo de cambio químico acorde con el científicamente aceptado.
LLORENS, J.A. (1991). Comenzando a aprender química. Madrid: Visor.

MARTÍN DEL POZO, R. (1994). El conocimiento del cambio químico en la formación inicial del profesorado. Estudio de las concepciones disciplinarias y didácticas de los estudiantes de magisterio. Tesis doctoral. Universidad de Sevilla.

OGBORN, J. (1996). Methaphorical Understandings and Scientific Ideas. International Journal of Science Education, 18(6), pp. 631-652.

OGBORN, J., KRESS, G., MARTINS, I. y McGILLICUDY, K. (1996). Explaining science. Londres: The Open University Press.

POZO, J.I. (1991). Procesos cognitivos en la comprensión de la ciencia: las ideas de los adolescentes sobre la química. Madrid: CIDE-MEC.

PRIETO, T. et al. (1993). Consistency in pupils' explanations about combustion. Third International Seminar Misconceptions 
and Educational Strategies in Science and Mathematics. Nueva York: Cornell University.

RAMSDEN, J.M. (1997). How does a context-based approach influence understanding of key chemical ideas at $16+$ ? International Journal of Science Education, 19(6), pp. 697-710.

ROLETTO, E. y PIACENZA, B. (1994). Faut-il construire le concept de substance? Aster, 18, pp. 63-74.

SANNMARTÍ, N., IZQUIERDO, M. y WATSON, R. (1995). The substantialisation of Properties in Pupils' Thinking and in the History of Science. Science Education, 4, pp. 349-369.

SOLOMONIDOU, C. (1991). Comment se representer les substances et leurs interactions? Tesis. París VII. UFR Didactique des disciplines. Specialité: Didactique de la chimie.

SOLSONA, N. (1995). The emergence of chemical phenomena Research in Science Education, II, pp. 235- 240. Tesalónica: Art of text.

SOLSONA, N. (1997). L'emergència de la interpretació dels fenòmens químics. Tesis doctoral (Publicación en microficha). Barcelona: Universitat Autònoma de Barcelona.

SOLSONA, N. e IZQUIERDO, M. (1998a). La construcción del concepto de cambio químico. Los modelos teóricos, un instrumento para su análisis, en Banet y de Pro (coord.). Investigación e innovación en la enseñanza de las ciencias, pp. 327-335. Murcia.

SOLSONA, N. e IZQUIERDO, M. (1998b). La conservación delelemento, unaideainexistente en el alumnado de secundaria. Alambique, 17, pp. 76-84.

STAVRIDOU, H. y SOLOMONIDOU, C. (1998) Conceptual reorganization and the construction of the chemical reaction concept during secondary education. Int. Journal Science Education, 20(2), pp. 205-221

THAGARD, P. (1992). Analogy, Explanation and Education. Journal of Research in Science Teaching, 29(6), pp. 537-544.

TREAGUST, D. et al. (1996). Using an analogical teaching approach to engenderconceptual change.InternationalJournal of Science Education, 18(2), pp. 213-230.

WATSON, R. et al. (1995). The Effect of Practical Work on Students' Understanding of combustion. Journal of Research in Science Teaching, 32(5), pp. 487-502.

WHITE, R.T. (1996). The link between the laboratory and learning. International Journal Science Education, 18(7), pp. 761-774

[Artículo recibido en septiembre de 1998 y aceptado en mayo de 1999.] 